\title{
Pattern Avoiding Ballot Paths and Finite Operator Calculus
}

\author{
Heinrich Niederhausen and Shaun Sullivan \\ Florida Atlantic University, Boca Raton, Florida
}

November 8, 2018

\begin{abstract}
Counting pattern avoiding ballot paths begins with a careful analysis of the pattern. Not the length, but the characteristics of the pattern are responsible for the difficulties in finding explicit solutions. Certain features, like overlap and difference in number of $\rightarrow$ and $\uparrow$ steps determine the solution of the recursion formula. If the recursion can be solved by a polynomial sequence, we apply the Finite Operator Calculus to find an explicit form of the solution in terms of binomial coefficients.

Keywords: Pattern avoidance, ballot path, Dyck path, Finite Operator Calculus, Umbral Calculus
\end{abstract}

\section{Introduction}

A ballot path stays weakly above the diagonal $y=x$, starts at the origin and takes steps from $\{\uparrow, \rightarrow\}$. A pattern is a finite string made from the same step set; it is also a path. Dyck paths are equivalent to ballot paths, taking steps from $\{\nearrow, \searrow\}$, staying weakly above the $x$-axis. Dyck paths containing $k$ strings of length 3 were discussed by E. Deutsch in [1]. One of the most recent papers on patterns of length 4 occurring $k$ times in Dyck paths was written by A. Sapounakis, I. Tasoulas, P. Tsikouras, Counting strings in Dyck paths, 2007, to appear in Discrete Mathematics [6]. The authors find generating functions for all 16 patterns in Dyck path returning to the $x$-axis. Returning to the $x$-axis at the end of the path has the advantage that going backwards through a path we find the reversed pattern exactly the same number of times (see Section 2). This reduces significantly the number of patterns under consideration.

For easier presentation we decided to talk about ballot instead of Dyck paths. We will look at paths that end at any point $(n, m)$ above or on the main diagonal. That will give us more cases to consider, because the path reversal bijection will be of no help in general. On the other hand, we will investigate pattern avoiding paths only $(k=0)$, but we will do this for patterns of general length. We use the Finite Operator Calculus approach, as developed by G.-C. Rota, D. Kahaner, and A. Odlyzko [5]. An interpretation of this theory in view of solving recursions is given in 4]. The Finite Operator Calculus works with polynomials; it applies to pattern avoiding ballot paths, because for many patterns we can find a polynomial sequence whose values enumerate all possible paths.

For convenience, we let $u=\uparrow$ and $r=\rightarrow$. Of course, avoiding the pattern $p$ may imply avoiding a pattern that contains $p$ twice in some overlapping form, like uruuru is contained twice in uruururuuru, but also twice in uruuruuru. This problem of overlaps makes it harder to find recursions. There are two properties of the patterns we avoid that determine the recurrence relations.

Definition 1 The bifix index of a pattern $p$ is the number of distinct nonempty patterns o such that $p$ that can be written in the form $p=o p^{\prime}$ and $p=p^{\prime \prime} o$ for $o, p^{\prime}, p^{\prime \prime} \in\{u, r\}^{*}$. If a pattern has bifix index 0, then we call it bifix-free.

The example above, uruuru, has bifix index 2. We restrict ourselves to bifix index 0 or 1 in this paper. However, there is a notable exception; a pattern of only right steps has a high bifix index. We can avoid this pattern with a prefixed up step, which makes the prefixed pattern bifix free. Another 
exception is a pattern of only up steps. We will begin in Section 2 with this case to explain our approach.

Definition 2 Let $d(p)$ be the number of u's minus the number of $r$ 's in the pattern $p$. The depth of $p$ is $\max \left\{d\left(p^{\prime}\right) \mid p=q p^{\prime}, q \in\{u, r\}^{*}\right\}$.

Intuitively, the depth is the maximum distance $p$ goes below the line $y=x$ when the end of $p$ is attached to the line $y=x$. Notice that since the empy pattern $\epsilon$ is in $\left\{p^{\prime} \mid p=q p^{\prime}\right\}$, the depth of a pattern is always a nonnegative integer.

If $a$ is the number of $r$ 's in $p$ and $c$ is the number of $u$ 's, then we say $p$ has dimensions $a \times c$.

We consider patterns of the following form:

1. $r^{a}$ and $u^{c}$ for any $a, c \geq 0$.

2. Bifix-free patterns of depth 0 and dimensions $a \times c$, with $a \geq c \geq 1$, and $a \geq 2$.

3. Patterns of depth 0 with bifix index 0 or 1 (with the same restrictions as above, and corresponding restriction about the $o p^{\prime}$ piece of $p=o p^{\prime} o$ ).

4. Patterns with depth at least 1 and bifix index 0 or 1 (with similar restrictions).

5. All patterns of length 4 .

\section{Lattice paths avoiding $r^{a}$ and $u^{c}$}

Definition 3 Let $s_{n}(m ; p, l)$ be the number of lattice paths from $(0,0)$ to $(n, m)$ staying weakly above the line $y=x-l$ avoiding the pattern $p \in\{\uparrow, \rightarrow\}^{*}$.

Note that if $l=0$ we count pattern avoiding ballot paths. A ballot path must start with an up step; if the pattern to avoid is $r^{a}$, we are really avoiding $u r^{a}$. In other words, $s_{n}\left(m ; r^{a}, 0\right)=s_{n}\left(m ; u r^{a}, 0\right)$. This means that the bifix-rich pattern $r^{a}$ can be replaced by the bifix-free pattern $u r^{a}$. We loose this property if $l>0$. However, the recursion

$$
s_{n}\left(m ; r^{a}, l\right)=s_{n-1}\left(m ; r^{a}, l\right)+s_{n}\left(m-1 ; r^{a}, l\right)-s_{n-a}\left(m-1 ; r^{a}, l\right)
$$

still holds for all $m \geq n>0$. Only the initial values change with $l, s_{n}\left(0 ; r^{a}, l\right)=1$ for $0 \leq n \leq$ $\min (a-1, l)$, and 0 else. The recursion says that we must subtract from the ballot recursion the paths reaching $(n, m)$ with exactly $a$ down steps at the end, and one up step in the beginning. These are counted by $s_{n-a}\left(m-1 ; r^{a}, l\right)$.

\begin{tabular}{r||rrrrrrrrrr}
$m$ & & & & & & & & & \\
4 & 1 & 5 & 15 & 35 & 65 & 101 & 135 & 155 & 152 & 112 \\
3 & 1 & 4 & 10 & 20 & 31 & 40 & 44 & 40 & 28 & 0 \\
2 & 1 & 3 & 6 & 10 & 12 & 12 & 10 & 6 & 0 & -16 \\
1 & 1 & 2 & 3 & 4 & 3 & 2 & 1 & 0 & -3 & -14 \\
0 & 1 & 1 & 1 & 1 & 0 & 0 & 0 & 0 & -3 & -11 \\
\hline \hline$n:$ & 0 & 1 & 2 & 3 & 4 & 5 & 6 & 7 & 8 & 9
\end{tabular}

The table shows the number of paths avoiding $r^{4}$ or $r r r r$ above the zeros and extending below the boundary using the recurrence to obtain a polynomial sequence.

Induction over $n$ shows that $\left(s_{n}\right)$ is a polynomial sequence with $\operatorname{deg} s_{n}=n$. Using operators on polynomials, we can write the recurrence as

$$
1-E^{-1}=B-B^{a} E^{-1}
$$


where the linear operators $B$ and $E^{v}$ are defined by linear extension of $B s_{n}(x)=s_{n-1}(x)$ and $E^{v} s_{n}(x)=s_{n}(x+v)$, the shift by $v$. The operators $\nabla=1-E^{-1}$ and $E^{-1}$ both have power series expansions in $D$, the derivative operator. Such operators are called shift-invariant. Hence $B$ must be shift-invariant too, and therefore commutes with $\nabla$ and $E^{v}$. The power series for $B$ must be of order 1 , because $B$ reduces degrees by 1 . Such linear operators are called delta operators. The basic sequence $\left(b_{n}(x)\right)_{n \geq 0}$ of a delta operator $B$ is a sequence of polynomials such that $\operatorname{deg} b_{n}=n$, $B b_{n}(x)=b_{n-1}(x)$ (like the Sheffer sequence $s_{n}(x)$ for $B$ ), and initial conditions $b_{n}(0)=\delta_{0, n}$ for all $n \in \mathbb{N}_{0}$. In our special case, the basic sequence is easily determined. Solving for $E^{1}$ shows that

$$
E^{1}=\sum_{i=0}^{a-1} B^{i}
$$

Finite Operator Calculus tells us [4, (2.5)] that if $E^{1}=1+\sigma(B)$, where $\sigma(t)$ is a power series of order 1 , then the basic sequence $b_{n}(x)$ of $B$ has the generating function

$$
\sum_{n \geq 0} b_{n}(x) t^{n}=(1+\sigma(t))^{x}
$$

Thus, in our case $b_{n}(x)=\left[t^{n}\right]\left(1+t+t^{2}+\cdots+t^{a-1}\right)^{x}$

Definition 4 The geometric coefficient is defined as

$$
\left(\begin{array}{l}
x \\
n
\end{array}\right)_{a}=\left[t^{n}\right]\left(1+t+\cdots+t^{a-1}\right)^{x}=\sum_{i=0}^{\lfloor n / a\rfloor}(-1)^{i}\left(\begin{array}{c}
x \\
i
\end{array}\right)\left(\begin{array}{c}
x+n-a i-1 \\
n-a i
\end{array}\right)
$$

Note that for $a=2$ the geometric coefficient equals the binomial coefficient $\left(\begin{array}{l}x \\ n\end{array}\right)$. These numbers have already been studied by Euler [2. Therefore they are also called Eulerian coefficients. Some interesting properties of geomeric coefficients are given in [3].

Thus $b_{n}(x)=\left(\begin{array}{l}x \\ n\end{array}\right)_{a}$. For $l=0$ the Sheffer sequence $\left(s_{n}\right)$ has initial values $s_{n}(n-1)=\delta_{n, 0}$. Abelization [4] gives us

$$
s_{n}\left(x ; r^{a}, 0\right)=\frac{x-n+1}{x+1} b_{n}(x+1)=\frac{x-n+1}{x+1}\left(\begin{array}{c}
x+1 \\
n
\end{array}\right)_{a}
$$

so the number of ballot paths avoiding $r^{a}$ is

$$
s_{n}\left(n ; r^{a}, 0\right)=\frac{1}{n+1}\left(\begin{array}{c}
n+1 \\
n
\end{array}\right)_{a} .
$$

The reflection of a pattern $p$ is the pattern where every up step becomes a right step, and vice versa. The pattern $\tilde{p}$ is the reverse of the pattern $p$, if it is the reflection of $p$ read backwards; for example if $p=$ uruuruu, then $\tilde{p}=$ rrurrur. It is a fundamental principal in pattern avoidance, proved by reflection, that the number of $\{\uparrow, \rightarrow\}$ lattice paths weakly above $y=x$ ending at $(n, n+l)$ avoiding $p$ equals the number of $\{\uparrow, \rightarrow\}$ lattice paths weakly above $y=x-l$ ending at $(n+l, l)$ avoiding the reverse pattern $\tilde{p}$,

$$
s_{n}(n+l ; p, 0)=s_{n+l}(n ; \tilde{p}, l) .
$$

If we are only interested in paths returning to the diagonal $(l=0)$, we can see how this principal saves us a great deal of work. For general $l>0$, this will not be the case, because we will not be able to find the paths avoiding $\tilde{p}$ staying weakly above $y=x-l$. There is one notable exception, $p=r^{a}$. For general $l$, the initial values $s_{n}\left(0 ; r^{a}, l\right)$ agree up to $n=l$ with $b_{n}(1)$, and therefore $s_{n}\left(x ; r^{a}, l\right)=b_{n}(x+1)$ for all $n \leq l$. For $n>l$ we have $s_{n}\left(n-l-1 ; r^{a}, l\right)=0$. The Binomial Theorem for Sheffer Sequences [4] expands $\left(s_{n}\right)$ under these and similar initial values. 
Theorem 5 If $\left(t_{n}\right)$ is a Sheffer sequence and $\left(q_{n}\right)$ the basic sequence for the same delta operator, then

$$
t_{n}(x+y)=\sum_{k=0}^{n} t_{k}(y+v k) \frac{x-v n}{x-v k} q_{n-k}(x-v k)
$$

for all $v \in \mathbb{R}$.

Hence

$$
\begin{aligned}
s_{n+l}\left(n ; r^{a}, l\right) & =\sum_{k=0}^{n+l} s_{k}\left(k-l-1 ; r^{a}, l\right) \frac{1}{n+l+1-k} b_{n+l-k}(n+l+1-k) \\
& =\sum_{k=0}^{l} \frac{1}{n+l+1-k}\left(\begin{array}{c}
k-l \\
k
\end{array}\right)_{a}\left(\begin{array}{c}
n+l+1-k \\
n+l-k
\end{array}\right)_{a} .
\end{aligned}
$$

Of course, this expansion reduces to (1) if $l=0$.

Because we were able to find the number of paths weakly above $y=x-l$ for general $l$ as polynomials, we are also able to apply the above general principle, saying that $s_{n}\left(n+l ; u^{c}, 0\right)=s_{n+l}\left(n ; r^{c}, l\right)$. Thus

$$
\begin{aligned}
& s_{n}\left(m ; u^{c}, 0\right)=\sum_{k=0}^{m-n} \frac{1}{m+1-k}\left(\begin{array}{c}
k-m+n \\
k
\end{array}\right)_{c}\left(\begin{array}{c}
m+1-k \\
m-k
\end{array}\right)_{c}
\end{aligned}
$$

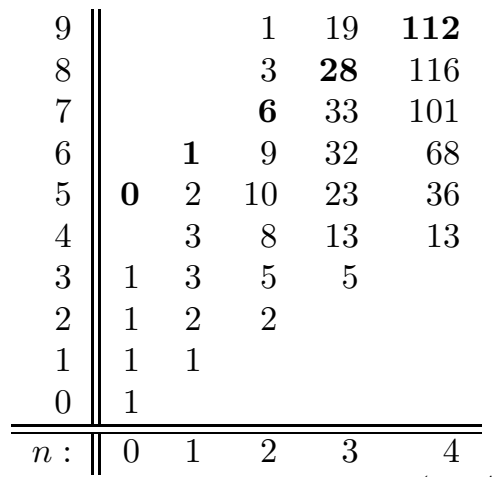

$$
\begin{aligned}
& s_{n}\left(m ; u^{4}, 0\right) \text { (the numbers in bold agree with } s_{n+5}\left(n ; r^{4}, 5\right) \text { in the Table above) }
\end{aligned}
$$

\section{Bifix-free patterns with depth 0}

From now on we will only look at paths weakly above the diagonal $y=x$. We write $s_{n}(x ; p)$ instead of $s_{n}(x ; p, 0)$, and we also may omit the pattern $p$ from the notation. As with the pattern $r^{a}$, we can find a single recurrence relation that holds everywhere in the octant. If the pattern is bifix-free, we need only to subtract paths that would end in the pattern, thus we have the recurrence

$$
s_{n}(m ; p)=s_{n-1}(m ; p)+s_{n}(m-1 ; p)-s_{n-a}(m-c ; p)
$$

where $p$ has dimensions $a \times c$. For example uurrurrur has dimensions $5 \times 4$, and depth 0 . The recurrence has a polynomial solution if the depth is 0 , and $a \geq c \geq 1, a \geq 2$ (If $a=1$ then $p=u r$, a pattern we do not want to avoid). In operators:

$$
\nabla=B\left(1-B^{a-1} E^{-c}\right)
$$

Since the delta operator $\nabla$ can be written as a delta series in $B$, the operator $B$ is also a delta operator. The basic sequence can be expressed via the Transfer Formula [4, Theorem 1]:

$$
b_{n}(x)=x \sum_{i=0}^{\frac{n}{a-1}} \frac{(-1)^{i}}{x-c i}\left(\begin{array}{c}
n-(a-1) i \\
i
\end{array}\right)\left(\begin{array}{c}
x+n-(a+c-1) i-1 \\
n-(a-1) i
\end{array}\right)
$$


Since our initial values are $s_{n}(n-1 ; p)=\delta_{n, 0}$, we use Abelization [4, (2.5)] to obtain:

$$
s_{n}(x)=(x-n+1) \sum_{i=0}^{\frac{n}{a-1}} \frac{(-1)^{i}}{x-c i+1}\left(\begin{array}{c}
n-(a-1) i \\
i
\end{array}\right)\left(\begin{array}{c}
x+n-(a+c-1) i \\
n-(a-1) i
\end{array}\right)
$$

Therefore the number of ballot paths avoiding $p$ and returning to the diagonal is

$$
s_{n}(n)=\sum_{i=0}^{\frac{n}{a-1}} \frac{(-1)^{i}}{n-c i+1}\left(\begin{array}{c}
n-(a-1) i \\
i
\end{array}\right)\left(\begin{array}{c}
2 n-(a+c-1) i \\
n-(a-1) i
\end{array}\right)
$$

\section{Patterns with depth 0 and bifix index 1}

The pattern $p$ has bifix index 1 if there exists a unique nonempty pattern $o$ such that $p=o p^{\prime} o$. If $p$ has dimensions $a \times c$ and $o p^{\prime}$ has dimensions $b \times d$, we have a recurrence of the form

$$
s_{n}(x)=s_{n-1}(x)+s_{n}(x-1)-\sum_{i \geq 0}(-1)^{i} s_{n-a-b i}(x-c-d i)
$$

For example let $p=$ urruurr. It has depth 0 , with dimensions $4 \times 3$ and bifix urr, so $b=2$ and $d=2$. From the paths reaching $(n-a, x-c)$ those ending in urru cannot be included in the recurrence and must be subtracted, and from those again we cannot include paths ending in urru, and so on. The $o p^{\prime}$ piece of the pattern that is responsible for this exclusion-inclusion process may not go below the diagonal; hence it must have depth $\leq a-c$.

From examining the summation in the recurrence, we notice that we must have $b \geq d$. If $b<d$, then at some point we would be using numbers below the $y=x$ boundary, which are only the polynomial extensions and do not count paths. Notice in our above example $b-d=0$, thus the recurrence formula applies to the pattern urruurr.

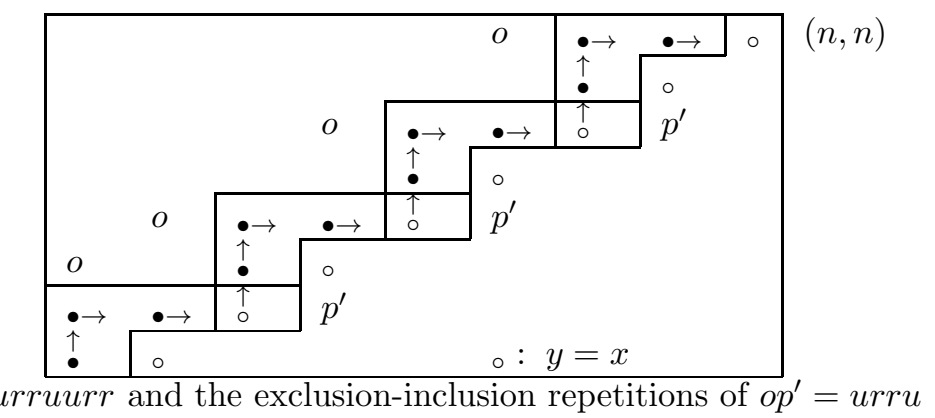

Summary of the conditions on $p$ :

1. $p=o p^{\prime} o$, where $o$ is nonempty and unique (bifix index $=1$ ).

2. $p$ has dimensions $a \times c$, where $a \geq c \geq 1, a \geq 1$.

3. $p^{\prime} o$ has dimensions $b \times d$, where $b \geq d, b \geq 1$.

4. $\operatorname{depth}\left(p^{\prime} o\right) \leq a-c$.

We assume that $b \geq 1$, because otherwise $p=u^{c}$, a case we considered already in a previous section. Under these conditions $\left(s_{n}\right)$ is a Sheffer sequence, and the recursion (3) can be written in terms of the operator $B: s_{n} \mapsto s_{n-1}$ as

$$
1=B+E^{-1}-\sum_{i \geq 0}(-1)^{i} B^{a+b i} E^{-c-d i}=B+E^{-1}-\frac{B^{a} E^{-c}}{1+B^{b} E^{-d}}
$$

or 


$$
\begin{aligned}
\nabla & =B+B^{b+1} E^{-d}+B^{b} E^{-d-1}-B^{a} E^{-c}-B^{b} E^{-d} \\
& =B-B^{a} E^{-c}+B^{b+1} E^{-d}-B^{b} E^{-d} \nabla
\end{aligned}
$$

We view $\nabla$ as a formal power series in $B$ with coefficients in the ring of shift-invariant operators. The generalized Transfer Formula 4, Theorem 2] tells us that

$$
b_{n}(x)=x \sum_{i=1}^{n} C_{n, i} \frac{1}{i}\left(\begin{array}{c}
i-1+x \\
i-1
\end{array}\right)
$$

is the basic polynomial for $B$, where

$$
C_{n, i}=\left[B^{n}\right]\left(B-B^{a} E^{-c}+B^{b+1} E^{-d}-B^{b} E^{-d} \nabla\right)^{i}
$$

Hence

$$
\begin{aligned}
b_{n}(x)= & x \sum_{j, k, l \geq 0:(a-1) j+b k+(b-1) l \leq n-1}\left(\begin{array}{c}
n-(a-1) j-b k-(b-1) l \\
j, k, l
\end{array}\right)(-1)^{j+l} \\
& \times E^{-c j-d k-d l} \nabla^{l} \frac{1}{n-(a-1) j-b k-(b-1) l}\left(\begin{array}{c}
n-(a-1) j-b k-(b-1) l-1+x \\
n-(a-1) j-b k-(b-1) l-1
\end{array}\right) \\
= & x \sum_{j=0}^{\frac{n-1}{a-1}} \sum_{k=0}^{\frac{n-1-(a-1) j}{b} \frac{n-1-(a-1) j}{b}-k}\left(\begin{array}{c}
n-(a-1) j-b k-(b-1) l \\
j, k, l
\end{array}\right) \\
& \times \frac{(-1)^{j+l}}{n-(a-1) j-b k-(b-1) l}\left(\begin{array}{c}
n-(a+c-1) j-(b+d)(k+l)-1+x \\
n-(a-1) j-b(k+l)-1
\end{array}\right)
\end{aligned}
$$

Because $s_{n}(n-1)=\delta_{0, n}$ we still get $s_{n}(x)=\frac{x-n+1}{x+1} b_{n}(x+1)$.

\begin{tabular}{l||llllllllll}
$m$ & 1 & 8 & 35 & 110 & 270 & 544 & 920 & 1272 & 1236 & 0 \\
7 & 1 & 7 & 27 & 75 & 161 & 279 & 389 & 377 & 0 & \\
6 & 1 & 6 & 20 & 48 & 87 & 122 & 118 & 0 & & \\
5 & 1 & 5 & 14 & 28 & 40 & 38 & 0 & & & \\
4 & 1 & 4 & 9 & 14 & 13 & 0 & & & & \\
3 & 1 & 3 & 5 & 5 & 0 & & & & & \\
2 & 1 & 2 & 2 & 0 & & & & & & \\
1 & 1 & 1 & 0 & & & & & & & \\
0 & 1 & 0 & & & & & & & & \\
\hline \hline & 0 & 1 & 2 & 3 & 4 & 5 & 6 & 7 & 8 & $n$
\end{tabular}

The number of ballot paths avoiding urruurr

Example 6 For the pattern urruurr we find the parameters $a=\# r^{\prime} s=4, c=\# u$ 's $=3, b=2$, $d=2$. Because the depth (urruurr $)=0$, we obtain the explicit solution

$s(n, x)=\sum_{j=0}^{\lfloor(n-1) / 3\rfloor} \sum_{k=0}^{\lfloor(n-1-3 j) / 2\rfloor} \sum_{l=0}^{\lfloor(n-1-3 j-2 k) / 2\rfloor}\left(\begin{array}{c}n-3 j-2 k-l \\ j\end{array}\right)\left(\begin{array}{c}n-4 j-2 k-l \\ k\end{array}\right)\left(\begin{array}{c}n-4 j-3 k-l \\ l\end{array}\right) \times$ $\times \frac{(x-n+1)(-1)^{j+l}}{n-3 j-2 k-l}\left(\begin{array}{l}n-6 j-4(k+l)+x \\ n-3 j-2(k+l)-1\end{array}\right)$

The above operator equation (44) simplifies when $a=b+1$ and $c=d$. This corresponds to a pattern of the form $r p^{\prime} r$. For this case we get

$$
\nabla=B\left(1+B^{b} E^{-d}\right)^{-1}
$$

and

$$
s_{n}(x)=\frac{(x-n+1}{x+1} b_{n}(x+1)=\sum_{i=0}^{\lfloor n / b\rfloor} \frac{(-1)^{i}}{x-d i+1}\left(\begin{array}{c}
n-(b-1) i-1 \\
i
\end{array}\right)\left(\begin{array}{c}
x+n-(d+b+1) i \\
n-b i
\end{array}\right)
$$


The number of paths returning to the diagonal equals

$$
s_{n}(n)=\sum_{i=0}^{\lfloor n / b\rfloor} \frac{(-1)^{i}}{n-d i+1}\left(\begin{array}{c}
n-(b-1) i-1 \\
i
\end{array}\right)\left(\begin{array}{c}
2 n-(d+b+1) i \\
n-b i
\end{array}\right) .
$$

\section{$5 \quad$ Patterns with depth at least 1 and bifix index 0 or 1}

Up to now we studied patterns such that the pattern avoiding ballot numbers could be continued to Sheffer polynomials below the diagonal, using the notation $s_{n}(x)$. Because that is no longer true in this Section, we begin with the new notation $D(n, m)$ for the pattern avoiding ballot numbers reaching $(n, m)$. It is easy to see that if the pattern has depth $\delta>0$, then the recurrence for all points $(n, m)$ between the lines $y=x$ and $y=x+\delta$ is

$$
D(n, m)=D(n-1, m)+D(n, m-1)
$$

since by definition of depth a path ending at $(n, m)$ with $n+\delta>m \geq n$ cannot contain the pattern $p$. In general, these numbers can not be extended to polynomials of degree $n$. As initial values we have $D(n, n-1)=\delta_{0, n}$ for all $n \geq 0$.

If $(n, m)$ falls weakly above the line $y=x+\delta$ we have the recurrence

$$
D(n, m)=D(n-1, m)+D(n, m-1)-D(n-a, m-c)
$$

for bifix index 0 , and

$$
D(n, m)=D(n-1, m)+D(n, m-1)-\sum_{i \geq 0}(-1)^{i} D(n-a-b i, m-c-d i)
$$

for bifix index 1 (see (2) and (3)).

We obtain a Sheffer sequence $\left(s_{n}\right)$, say, but only weakly above the line $y=x+\delta$,

$$
D(n, m)=s_{n}(m) \text { for all } m \geq \delta,
$$

if the correction terms $D(n-a, m-c)$ (or $\left.\sum_{i \geq 0}(-1)^{i} D(n-a-b i, m-c-d i)\right)$ in (5) or (6) are taken at points weakly above the same line, thus $a \geq c$ and $b \geq d$. Finally, we must enforce in case of a nonempty bifix $o$ that depth $\left(o p^{\prime}\right)$, where $p=o p^{\prime} o$, does not exceed $d+a-c$.

Looking closer at the boundary $m=n+\delta$,

$$
s_{n}(n+\delta)=s_{n-1}(n+\delta)+s_{n}(n+\delta-1)-\sum_{i \geq 0}(-1)^{i} s_{n-a-b i}(n+\delta-c-d i)
$$

we see that $s_{n}(n+\delta-1)$ and $D(n, n+\delta-1)$ also agree. We utilized this fact in the previous Sections to determine the initial values $s_{n}(n-1)=\delta_{0, n}$ when the depth was 0 .

Example 7 The following Table shows the number of ballot paths avoiding the pattern rrruuurrruu, which has depth $\delta=2, a-c=1$, one bifix rrruu, op $=$ rrruuu of depth 3 , and $b-d=0$. 


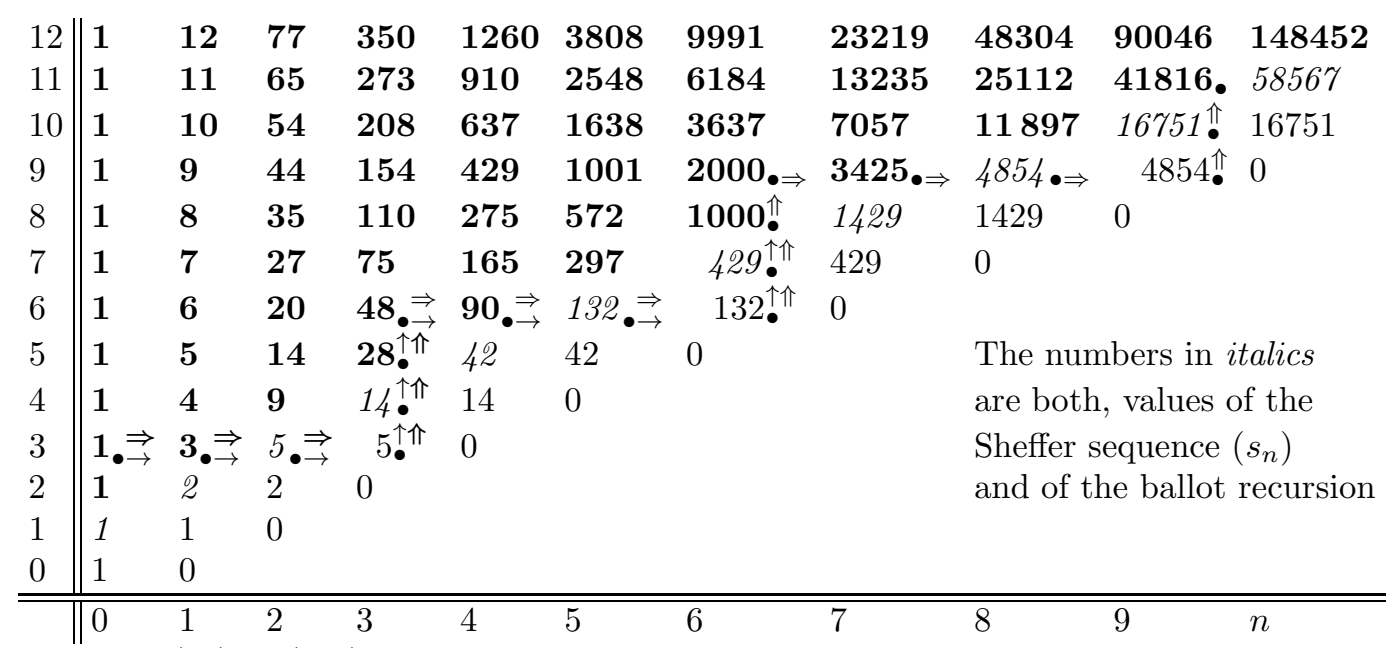

The path $(\rightarrow)$ to $(6,8)$ shows the first instance when the occurrence of the pattern is subtracted. The double path $(\Rightarrow)$ to $(9,11)$ shows the first instance when the occurrence of the $o p^{\prime}$ path is added.

How can we determine initial values for the Sheffer sequence $\left(s_{n}\right)$ if $\delta>0$ ? There are several ways of doing this; one way applies the following Lemma to obtain a (recursive) formula for the values $s_{n}(n+\delta-1)$. Such a recursion will be enough for explicitly representing the Sheffer polynomials in terms of their basic sequence.

Lemma 8 Let $a=\# r$ 's in $p$ be larger than 1 . For all depths $\delta \geq 0$ holds $s_{0}(x)=1$, and for $0 \leq j \leq \delta$ holds

$D(n, n+j-1)=\sum_{i=1}^{j / 2}\left(\begin{array}{c}j-i \\ i\end{array}\right)(-1)^{i-1} D(n-i, n-i+j-1)+\sum_{i=1}^{(j+1) / 2}\left(\begin{array}{c}j-i \\ i-1\end{array}\right)(-1)^{i-1} D(n-i, n-i+j)$

for all $n \geq 1$. Especially

$$
s_{n}(n+\delta-1)=\sum_{i=1}^{\delta / 2}\left(\begin{array}{c}
\delta-i \\
i
\end{array}\right)(-1)^{i-1} s_{n-i}(n-i+\delta-1)+\sum_{i=1}^{(\delta+1) / 2}\left(\begin{array}{c}
\delta-i \\
i-1
\end{array}\right)(-1)^{i-1} s_{n-i}(n-i+\delta)
$$

Proof. For $n=1$ we get

$$
D(1, j)=\left(\begin{array}{c}
j-1 \\
1
\end{array}\right) D(0, j-1)+\left(\begin{array}{c}
j-1 \\
0
\end{array}\right) D(0, j)=j
$$

which is right because $a>1$. For $j=0$ we get $D(n, n-1)=0$ as required. The double induction over $n \geq 1$ and $1 \leq j \leq \delta$ uses only the recursion $D(n, n-1+j)=D(n-1, n-1+j)+D(n, n-2+j)$.

Example 9 (continued) In the example avoiding the pattern rrruuurruu, which has $\delta=2$, we find $s_{n}(n+1)=s_{n-1}(n)+s_{n-1}(n+1)$.

Using the above initial values from Lemma 8 to find $s_{n}(x)$ above the line $y=x+\delta$ requires the Functional Expansion Theorem ([4, Theorem 3]):

Theorem 10 Suppose $\left(s_{n}\right)$ is a Sheffer sequence for the delta operator $B$ with basic sequence $\left(b_{n}\right)$, and $L$ a functional such that $\langle L \mid 1\rangle \neq 0$. Then

$$
s_{n}(x)=\sum_{k=0}^{n}\left\langle L \mid s_{k}\right\rangle \tilde{L}^{-1} b_{n-k}(x)
$$

where $\tilde{L}^{-1}$ is the operator inverse to $\tilde{L}=\sum_{i \geq 0}\left\langle L \mid b_{i}\right\rangle B^{i}$. 
Lemma 8 tells us how to choose the functional $L$,

$$
\begin{aligned}
& \left\langle L \mid s_{n}(n+x)\right\rangle \\
= & s_{n}(n+\delta-1)-\sum_{i=1}^{\delta / 2}\left(\begin{array}{c}
\delta-i \\
i
\end{array}\right)(-1)^{i-1} s_{n-i}(n-i+\delta-1)-\sum_{i=1}^{(\delta+1) / 2}\left(\begin{array}{c}
\delta-i \\
i-1
\end{array}\right)(-1)^{i-1} s_{n-i}(n-i+\delta) \\
= & \delta_{0, n}
\end{aligned}
$$

Note that we applied the functional $L$ to $s_{n}(n+x)$, a Sheffer polynomial for the delta operator $B_{1}:=E^{-1} B$, with basic polynomials $(x+n) b_{n}(x) / x$ (see [4, Remark 1]). In terms of the evaluation functional $\operatorname{Eval}_{z} f(x)=f(z)$ we can write

$$
\begin{aligned}
\left\langle L \mid s_{n}(n+x)\right\rangle= & \left(\operatorname{Eval}_{\delta-1}-\operatorname{Eval}_{\delta-1} \sum_{i=1}^{\delta / 2}\left(\begin{array}{c}
\delta-i \\
i
\end{array}\right)(-1)^{i-1} B_{1}^{i}\right. \\
& \left.-\operatorname{Eval}_{\delta} \sum_{i=1}^{(\delta+1) / 2}\left(\begin{array}{c}
\delta-i \\
i-1
\end{array}\right)(-1)^{i-1} B_{1}^{i}\right) s_{n}(n+x)
\end{aligned}
$$

According to [4, (2.7)] we get

$$
\tilde{L}=E^{\delta-1}-E^{\delta-1} \sum_{i=1}^{\delta / 2}\left(\begin{array}{c}
\delta-i \\
i
\end{array}\right)(-1)^{i-1} B_{1}^{i}-E^{\delta} \sum_{i=1}^{(\delta+1) / 2}\left(\begin{array}{c}
\delta-i \\
i-1
\end{array}\right)(-1)^{i-1} B_{1}^{i}
$$

and finally

$$
\tilde{L}^{-1}=E^{1-\delta} /\left(1-\sum_{i=1}^{(\delta+1) / 2}\left(\left(\begin{array}{c}
\delta-i \\
i
\end{array}\right)+E^{1}\left(\begin{array}{c}
\delta-i \\
i-1
\end{array}\right)\right)(-1)^{i-1} B_{1}^{i}\right)
$$

Therefore, the Functional Expansion Theorem writes $s_{n}(x)$ in terms of $b_{n}(x)$ as

$$
\begin{aligned}
s_{n}(n+x)= & \tilde{L}^{-1} \frac{x}{n+x} b_{n}(n+x) \\
= & \frac{E^{1-\delta}}{1+\sum_{i=1}^{(\delta+1) / 2}\left(\left(\begin{array}{c}
\delta-i \\
i
\end{array}\right)+E^{1}\left(\begin{array}{c}
\delta-i \\
i-1
\end{array}\right)\right)(-1)^{i} B_{1}^{i}} \frac{x}{n+x} b_{n}(n+x) \\
= & \sum_{j \geq 0}(-1)^{j}\left(\sum_{i=1}^{(\delta+1) / 2}\left(\left(\begin{array}{c}
\delta-i \\
i
\end{array}\right)+E^{1}\left(\begin{array}{c}
\delta-i \\
i-1
\end{array}\right)\right)(-1)^{i} B_{1}^{i}\right)^{j} \frac{x+1-\delta}{n+x+1-\delta} b_{n}(n+x+1-\delta)
\end{aligned}
$$

We leave the final expansion to the reader. Note that $B_{1}^{k} \frac{x+\delta-1}{n+x+\delta-1} b_{n}(n+x+1-\delta)=\frac{x+\delta-1}{n-k+x+\delta-1} b_{n-k}(n-k+x+1-\delta)$. Of course, thevalues $s_{n}(n+x)$ agree with the pattern avoiding path counts only for $x \geq \delta-1$.

Example 11 (continued) In the example above, avoiding the pattern rrruuurrruu, which has $\delta=2$, we find

$$
\begin{aligned}
s_{n}(n+x) & =\sum_{j \geq 0}\left(1+E^{1}\right)^{j} B_{1}^{j} \frac{x-1}{n+x-1} b_{n}(n+x-1) \\
& =2^{n}+\sum_{j=1}^{n} \sum_{i=0}^{n-j}\left(\begin{array}{c}
n-j \\
i
\end{array}\right) \frac{x+i-1}{j+x+i-1} b_{j}(j+x+i-1) .
\end{aligned}
$$

Finally, we also have to expand the ballot like numbers between the lines $y=x$ and $y=x+\delta$ in terms of those weakly above the line $y=x+\delta-1$. 
Lemma 12 We have for all $0 \leq j \leq \delta-1$

$$
D(n, n+\delta-1-j)=\sum_{i=0}^{j / 2}\left(\begin{array}{c}
j-i \\
i
\end{array}\right)(-1)^{i} s_{n-i}(n-i+\delta-1)+\sum_{i=1}^{(j+1) / 2}\left(\begin{array}{c}
j-i \\
i-1
\end{array}\right)(-1)^{i} s_{n-i}(n-i+\delta) .
$$

Proof. Similar to the proof of Lemma 8

Example 13 In the above example, avoiding the pattern rrruuurrruu, we have $\delta=2$. We find $D(n, n+1-j)=s_{n}(n+1)-\left(\begin{array}{c}j-1 \\ 0\end{array}\right) s_{n-1}(n+1)$ for $j=1,2$. For example, $D(10,10)=s_{10}(11)-$ $s_{9}(11)=58572-41821=16751$.

\section{The patterns of length 4}

In their paper Counting Strings in Dyck Paths [6], A. Sapounakis, I. Tasoulas, and P. Tsikouras find generating functions for all patterns of length 4 occurring $k$ times in Dyck paths, i.e., in ballot paths returning to the diagonal. Their case $k=0$ is our pattern avoiding case. All patterns of length four are included in the above considerations, except uuur, uuru, uruu, and ruuu. The path counts in these four cases are not (eventually) Sheffer polynomial; the Finite Operator Calculus does not directly apply.

ruuu After differencing, this case is similar to $u^{3}$, and we obtain for $m \geq 1$

$$
D\left(n, n+m ; r u^{3}\right)=\left(\begin{array}{l}
n \\
n
\end{array}\right)_{3}+\left(\begin{array}{c}
n \\
n-m
\end{array}\right)_{3}+2 \sum_{i=1}^{m-1}\left(\begin{array}{c}
n \\
n-i
\end{array}\right)_{3}
$$

Note that $D\left(n, n ; r u^{3}\right)=D\left(n-1, n ; r u^{3}\right)$.

uuur In a similar way as in the case $r u^{3}$, we obtain

$$
D\left(n, n+m ; u^{3} r\right)=\sum_{i=0}^{m} \sum_{k=0}^{i+1} \frac{1}{n+i+1-k}\left(\begin{array}{c}
k-i-1 \\
k
\end{array}\right)_{3}\left(\begin{array}{c}
n+i+1-k \\
n+i-k
\end{array}\right)_{3}
$$

which also holds for $m=0$.

uuru and uruu Both cases are avoided by the same ballot paths. We obtain $D(n, n+l ;$ uuru $)=D(n, n+l ;$ uruu $)$

$$
\begin{aligned}
= & \sum_{k=0}^{n}\left(\begin{array}{c}
m-1 \\
n-k
\end{array}\right)(-1)^{n-k} \sum_{i=0}^{k / 2}\left(\begin{array}{c}
2 i-k-1 \\
i
\end{array}\right) \frac{1}{k+1-2 i} \times \\
& \times\left(m\left(\begin{array}{c}
2 k+m-3 i \\
k-2 i-1
\end{array}\right)+\left(\begin{array}{c}
2 k+m-3 i \\
k-2 i
\end{array}\right)\right)
\end{aligned}
$$

Note that except for the special cases $u^{4}$ and $r^{4}$ no pattern of length 4 has a bifix index larger than 1.

\section{References}

[1] Deutsch, E., 1999. Dyck path enumeration. Discrete Math. 204, 167 - 202.

[2] Euler, L., 1801. De evolutione potestatis polynomialis cuiuscunque $\left(1+x+x^{2}+x^{3}+x^{4}+e t c .\right)^{n}$. Nova Acta Academiae Scientarum Imperialis Petropolitinae 12, 47 - 57.

[3] Niederhausen, H., Sullivan, S., 2007. Euler Coefficients and Restricted Dyck Paths. To appear in Congr. Enumerantium 2007 (arXiv:0705.3065).

[4] Niederhausen, H., 2003. Rota's umbral calculus and recursions. Algebra Univers. 49, 435 - 457. 
[5] Rota, G.-C., Kahaner, D., Odlyzko, A., 1973. On the Foundations of Combinatorial Theory VIII: Finite operator calculus. J. Math. Anal. Appl. 42, 684- 760.

[6] Sapounakis, A., Tasoulas, I., Tsikouras, P., 2007. Counting strings in Dyck paths. To appear in Discrete Mathematics. 